\title{
The Research of Disease Detection Method of Greenhouse Cucumber Leaf Based on Chlorophyll Fluorescence Analysis
}

\author{
R.K. Chang ${ }^{1, *}$, Y.H.Wang ${ }^{2}$, X.T. Zhang ${ }^{1}$, G.C.Tang ${ }^{1}$,Y.Wei ${ }^{1}$ \\ ${ }^{1}$ College of Engineering and Technology, Tianjin Agricultural University,. China \\ ${ }^{2}$ College of Horticulture and Landscape, Tianjin Agricultural University, China
}

Copyright @ 2015 Horizon Research Publishing All rights reserved.

\begin{abstract}
Currently, the plant physiological information detection technology and diagnostic systems has gradually become a hot topic in the research of facilities crop disease warning. By collecting the relevant data of changes in growth conditions of cucumber by Junior-PAM of CI-340 portable photosynthesis system, the experiment studied the correlation between leaf chlorophyll content and Photosynthetic rate, nutritional status and we like to search for the relationship between the changes in disease infection and chlorophyll content in the growth process of leaf. The experiment used different modeling methods to establish the relational model between fluorescence data such as FO ', FM', $\mathrm{FV} / \mathrm{FM}, \mathrm{FO}, \mathrm{FM}$ and SPAD, and then make predictions. The results show that the prediction of harmful levels of disease employing neural network model is the best with its MAE being 0.0025 and the accuracy up to $100 \%$.
\end{abstract}

Keywords Chlorophyll, Junior-PAM, Photosynthetic rate, SPAD Values

\section{Introduction}

As an important part in plant pathology, plant leaves diseases is drawing more and more attention of botanists and pathologist, because the disease can not only advance warning of plant growth, but also at the same time bring great help to the production of agriculture ${ }^{[1]}$. Present detection method of plant disease depends on visual method determining disease types through macroscopic observation of the experienced farmers and agriculture experts, which is subjective without the support of scientific and objective data. By studying correlation among the plant leaf chlorophyll content, photosynthetic rate and nutritional status as well as collecting the related data in plant growth condition, the experiment intends to explore the relationship between leaf disease infection and chlorophyll content in the process of plant growth so as to make the right prediction for the plant growth trend and lay the foundation for rapid detection of plant diseases devices.

Light energy utilized in the photosynthesis process of plants is absorbed through the chloroplast pigment (photosynthetic pigment). Chlorophyll content of plant leaf is closely related with photosynthetic rate and nutritional status. In general, plant growth condition is not only shown by detecting the chlorophyll content, but observing leaf color changes is also one of the important indices for seedlings diagnosis and water management. Common chlorophyll content detecting methods include spectrophotometry, living chlorophyll meter method and photoacoustic spectrum method ${ }^{[2-4]}$. Frequently applied method is Arnon method put forward by Mackinney and the early 80 's ethanol extraction method as well as acetone ethanol mixed method to extract the leaf turbulence. All of these chemical methods adopt solvent extraction method which not only destroy the integrity of the blade but are confined to detecting the absorption of the solution system, and require a series of calibration and compensation. Therefore, these methods with poor accuracy and complex operation, is not suitable for direct measurement of the crops.

Experimental design measured chlorophyll content indirectly with fluorescence spectroscopy ${ }^{[5]}$. Making use of the apparatus component Junior - PAM of CI-340 portable photosynthetic, and in the condition of blades dark adaptation, the experiment measured the biggest quantum efficiency of chlorophyll fluorescence parameters $\mathrm{Fv} / \mathrm{Fm}=$ (Fm-Fo)/Fm: PSII as well as the mixed Fo and maximum fluorescence Fm; then under light condition, the experiment detected the maximum fluorescence yield Fm', when hlorophyll fluorescence parameters was performing a saturated pulse PSII and reaction center was under the fully closed position, and the minimum fluorescence yield $\mathrm{Fo}^{\prime}$ when the PSII reaction center was in the open condition ${ }^{[6-8]}$. And then chlorophyll content from the same period was measured, the transformation curve model was fit out using the matlab software. Finally the content of chlorophyll was obtained by measuring fluorescence parameters of plants to determine the plant growth trend. 


\section{Experiments and Methods}

The Experimental Sample Preparation

With cucumber seedlings as the research object, the experiment used chlorophyll fluorescence spectra to detect plant physiology information, It's shown in Figure 1. The experiment chose Jinchun 4 of cucumber seeds from Kerun cucumber research institute of Tianjin agricultural university, cultivated cucumber sample in the laboratory of Tianjin agricultural university agronomy courtyard gardening, and tracked various physiological information in the process of cucumber leaf growth and chlorophyll fluorescence spectral data from March to May.

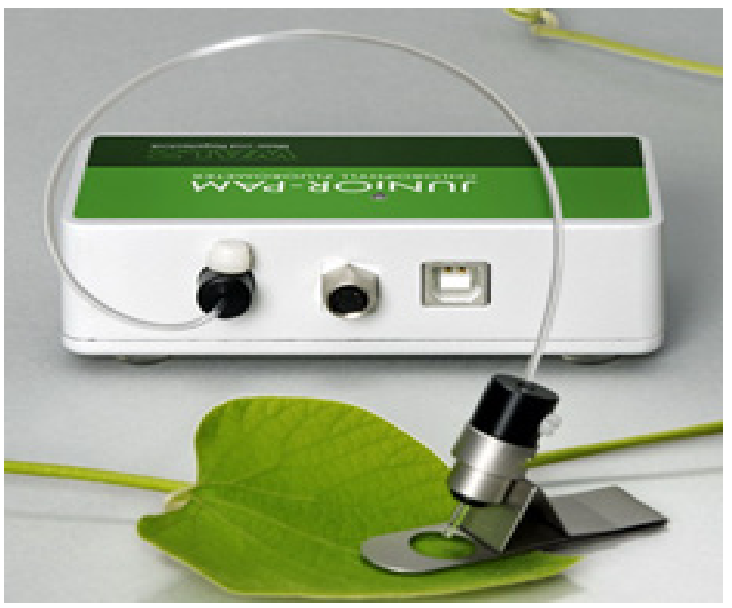

Figure 1. Real figure of Junior-PAM

\section{Data Collection}

Experiment firstly employed Junior-PAM instrument for the blade dark adaptation for 30 minutes, then put the blade in the magnetic clip on experiment, and then measured Fv/Fm fluorescence parameters by adjusting the measuring light intensity to ensure the real-time fluorescent $(\mathrm{Ft})$ is less than 600. But there are two problems in the measurement of plant leaf chlorophyll fluorescence parameters: (1) the instrument collected just a point of fluorescence parameters, which can't represent the whole blade; (2) As each sample has several blades, how can the fluorescence parameters of the whole sample be shown. Therefore, the experiment adopts the following methods in collecting and filtering the data.

(1) When measuring the samples, the experiment selected blades in good growth condition that can represent the culture medium of blades;

(2) Lord single blade veins middle position and two points on the edge of the blade, these three testing points of samples with dark adaptation for 20 minutes was selected for data collection.

(3) The experiment took average of each blade data collected and fluorescence parameter values to ensure the data to be representative ${ }^{[9]}$;

(4) Using matlab to look at the collected data to make a scatterplot, a big deviation will eliminate the data.

\section{Modeling and Orecasting}

The experiment picked out the five independent variables which have a significant impact on the parameters, such as Fo, Fm, Fv / Fm, Fo ', Fm' to establish partial least squares and neural network models. The Disease data at different stages of plant growth shows as table1.

Table 1. The Disease data at different stages of plant growth

\begin{tabular}{|c|c|c|c|c|c|c|}
\hline the stage of infection & SPAD & Fo & Fm & Fv/Fm & Fo' & Fm' \\
\hline normal growing infestation & $41.9-49.8$ & $280-358$ & $1290-1746$ & $0.77-0.80$ & $1293-1807$ & $286-363$ \\
\hline the initial infection & $36.1-40.2$ & $225-326$ & $750-1618$ & $0.77-0.82$ & $896-1451$ & $192-374$ \\
\hline the medium infection & $20.13-34.6$ & $289-367$ & $1322-1664$ & $0.75-0.78$ & $1248-1556$ & $308-373$ \\
\hline the late stage of infection & $9.3-19.5$ & $259-278$ & $1108-1140$ & $0.74-0.77$ & $986-1115$ & $261-299$ \\
\hline
\end{tabular}

Table 2. The correlation coefficient matrix

\begin{tabular}{|c|c|c|c|c|c|c|}
\hline & Fo & Fm & Fv/Fm & Fo' & Fm' & SPAD \\
\hline Fo & 1.0000 & 0.9576 & -0.0835 & 0.9329 & 0.9759 & 0.7735 \\
\hline Fm & 0.9576 & 1.0000 & 0.2063 & 0.9827 & 0.9495 & 0.9226 \\
\hline Fv/Fm & -0.0835 & 0.2063 & 1.0000 & 0.2433 & -0.0151 & 0.5670 \\
\hline Fo' & 0.9329 & 0.9827 & 0.2433 & 1.0000 & 0.9653 & 0.9273 \\
\hline Fm' & 0.9759 & 0.9495 & -0.0151 & 0.9653 & 1.0000 & 0.7990 \\
\hline SPAD & 0.7735 & 0.9226 & 0.5670 & 0.9273 & 0.7990 & 1.0000 \\
\hline
\end{tabular}




\section{Partial Least Squares Prediction and Error Data}

Partial least squares regression (PLS), applicable to multivariate data analysis, has a "response" matrix that can be extracted to reflect the maximum information of data variability. Hence, PLS can avoid not only non-normally distributed data, factor structure indeterminacy, but also potential problems such as unidentification of the model; in addition, it has a predictive function.

By calculating the data collected, the data correlation matrix could be obtained as shown in Table 2 .

The table 1 shows that there is positive correlation between Fo, Fv/Fm, Fo', Fm'and SPAD value; and there is negative correlation between Fo, Fm' and Fv/Fm.

Based on matlab programming and the respective score of each ingredient, four main ingredients are put forward to set partial least-squares regression model. The regression equation is shown in formula 1.

$$
y=-30.5653+0.0037 \times 1+0.0066 \times 2+78.2566 \times 3+0.0011 \times 4-0.0
$$

In order to investigate the accuracy of the model regression equation, in $\left(\tilde{y}_{i}, y_{i}\right)$, for the coordinate values, prediction diagram is made for all sample points as shown in Figure 2. $\tilde{y}_{i}$ is the k-th predictor variables. From Figure 1, all the points are evenly distributed along the diagonal graph, which shows that the difference is small between equation fitting values and the original value, thus this equation has the best fitting effect.

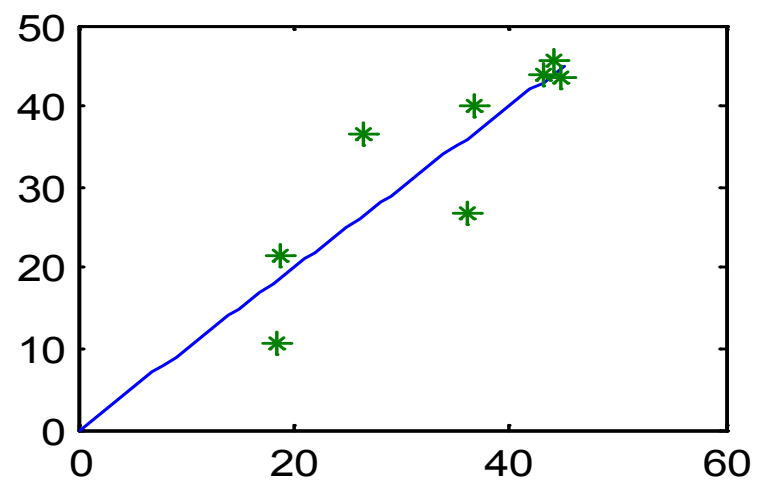

Figure 2. Relationship between predicted value and the actual value

After calculating the mean absolute error (MAE) is 4.725, the average error is 0.306 . Therefore, it can be seen that regression equation got through the partial least squares prediction is more accurate.

\section{BP Neural Network}

The experiment constructed four layer neural network of cucumber seedling leaves, Fo, Fm, Fv/Fm, Fo ', Fm as input, SPAD value of leaves as output ${ }^{[10-12]}$. After the test, the first hidden layer node number is 20 , the second hidden layer nodes is 40 , the number of training is 5000 , the error precision is set to: $1 * \mathrm{e}-6$, and the learning rate is 0.01 . Its network model and training error curve is shown in Figure 3and 4 .

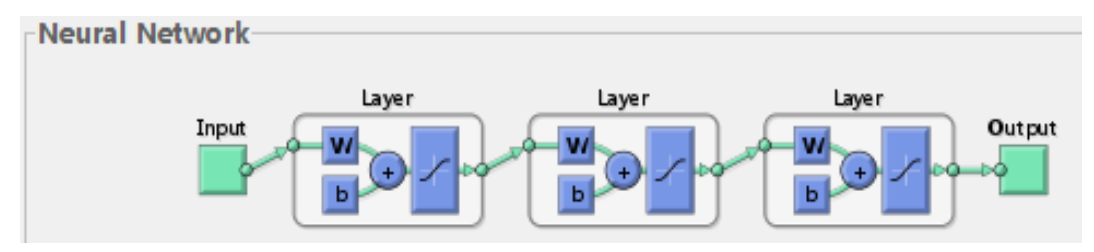

Figure 3. Neural Network Model

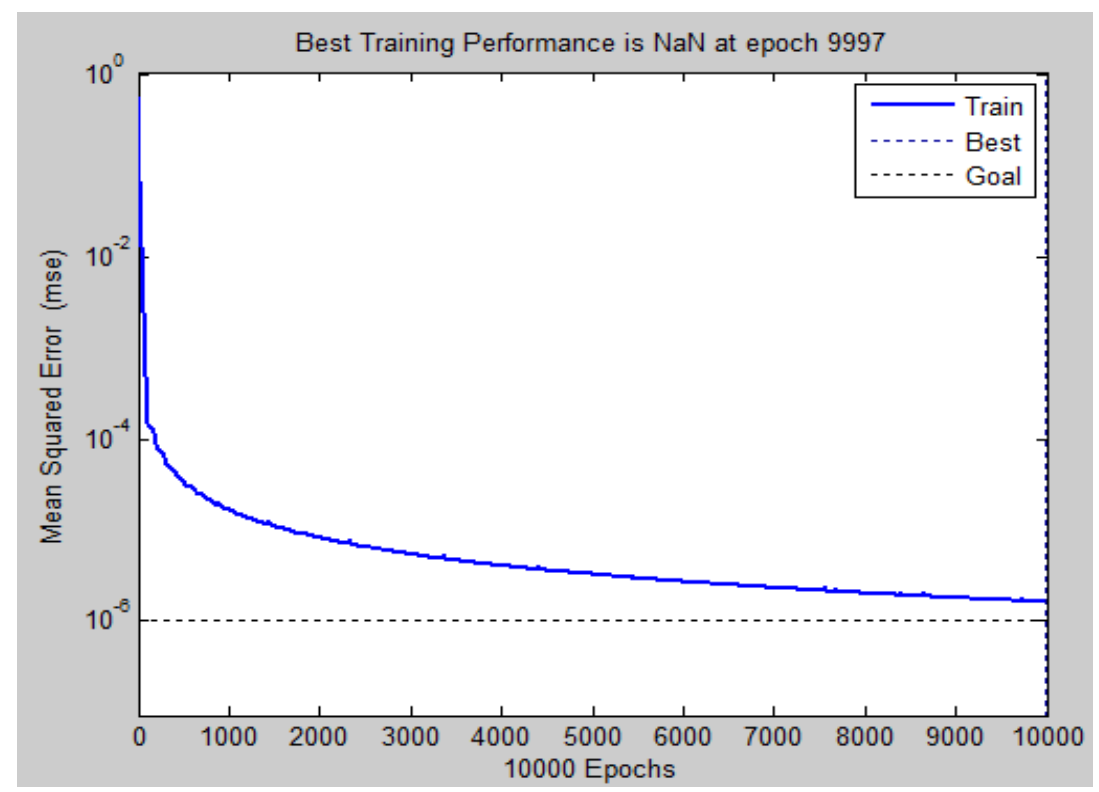

Figure 4. Error curve 
Table 3. SPAD predicted value and actual value and error

\begin{tabular}{|c|c|c|c|c|c|c|c|c|}
\hline Sample & 1 & 2 & 3 & 4 & 5 & 6 & 7 & 8 \\
\hline Actual value & 45.46 & 48.275 & 41.9 & 35.92 & 36.4 & 32.38 & 29.58 & 17.5 \\
\hline Predicted values & 45.4650 & 48.0484 & 41.9009 & 35.9201 & 36.4013 & 32.3823 & 29.5803 & 17.5161 \\
\hline error & -0.0050 & 0.22663 & -0.0008 & -0.0001 & -0.0012 & -0.0023 & -0.0003 & -0.0160 \\
\hline MAE & \multicolumn{7}{|c|}{0.0315725} \\
\hline
\end{tabular}

Table 4. Spectral data and SPAD value table

\begin{tabular}{|c|c|c|c|c|}
\hline & $\begin{array}{c}\text { normal growing } \\
\text { infestation 5.6 }\end{array}$ & $\begin{array}{c}\text { the initial } \\
\text { infection 5.15 }\end{array}$ & $\begin{array}{c}\text { The medium } \\
\text { infection 5.20 }\end{array}$ & $\begin{array}{c}\text { The late stage of infection } \\
5.22\end{array}$ \\
\hline fo & 315.6 & 227.25 & 296.75 & 145.5 \\
\hline fm & 1495 & 922.25 & 1092.50 & 575.25 \\
\hline Fv/fm & 0.78805 & 0.7535 & 0.71 & 0.74375 \\
\hline Fo' & 1392.2 & 946.75 & 1090.25 & 762.75 \\
\hline Fm' & 292.6 & 234.5 & 235 & 194.5 \\
\hline Actual SPAD value & 43.88 & 36.58 & 21.5 & 10.74 \\
\hline Predicted SPAD values & 43.8810 & 36.5799 & 21.5002, & 10.7836 \\
\hline
\end{tabular}

From table 3, it can be seen that with the application of neural network algorithm, the maximum of MAE between predicted values and actual values of is 0.0315725 .

Data analysis found that the MAE is up to 5.353 between the predicted and actual values by using partial least squares algorithm, but the maximum average error is only 0.606 , this phenomenon needs to be solved by increasing the sample data; And the maximum error is 0.2266 between the predictive value and actual value by using BP neural network algorithm, and the mean absolute error MAE maximum is 0.03157 , which can reach the expected value of SPAD, so the neural network is employed to predict SPAD values of different stages.

\section{Levels of Powdery Mildew in Cumber Leaves Scaling and Disease Detection}

For scaling of cucumber leaf disease infection degree, the experiment vaccinated virus on the cucumber growth. At the same time with reference to plant protection expert opinion[13], growth of cucumber leaf and disease infection degree were divided into normal growing degree of infestation, the initial infection, the medium and late stage of infection.

From table 4 , the maximum errors is 0.0436 between the SPAD value and actual value, the leaf growth conditions forecast reaching $100 \%$.

In summary, processing fluorescence spectral data of cucumber leaves with BP neural network to predict leaf SPAD values, solves such problems as the ordinary SPAD instruments can only measure single point which can not represent the whole leaves, and the chemical method destroys the integrity of the blade structure. As to prediction of the severity of lesion area, although the disease classification is the fuzzy region based on expert experience to determine, there is still some reference value.

\section{Conclusions}

From cucumber seedling stage, Experiment began to track detecting cucumber plant fluorescence spectral data and the SPAD value: (1) the experiment vaccinated virus after the cucumber leaves grew and conducted scaling of cucumber leaf disease infection degree: recording growth condition of cucumber leaf from normal growing degree of infestation, the initial infection, the medium and late stage of infection, and through testing the SPAD value made scaling of blades infection degree; (2) Select the optimal model to predict the growth of the plant state. By using partial least squares, the BP neural network algorithm and the like for fluorescence spectrum data such as Fo, Fm, Fv/Fm, Fo, Fm 'and' SPAD value, the experiment searched for the relationship between the fluorescence spectral data and the SPAD value. Through the comparison, neural network model was regarded as the optimal. Using the neural network model to predict disease harm, and then determine the state of plant growth, could ensure $100 \%$ accuracy.

In the process of plant growth,(1) only at the beginning, nutrient solution was added and after watering, no more nutrient solution was added, which can lead to the result that nutrient deficiency affects further growth at later growth stage of the plant;(2) In the inspection of prediction models, due to the limited data samples, the model has some limitations;(3) Fuzzy scaling of disease infection employed artificial observation, which still needs expert experience, is not conducive to promoting.

\section{Acknowledgements}

This research was performed with financial support from 
National Natural Science Foundation of China under the project No.31171892, College students of science and technology innovation projects of Tianjin Agricultural University, Innovation and Entrepreneurship Training Program of Tianjin Agricultural University under Grant No. 201410061191.

\section{Conflict of Interests}

We declare that we have no financial and personal relationships with other people or organizations.

\section{REFERENCES}

[1] C.K.PAI ,Acta Phytopathologica Sinica, 1957,3(2):137-154.

[2] M. W.Yang, Chinese Journal of Spectroscopy Laboratory, 2002,7,19:478-479.

[3] Z.S.Su, Plant Physiology Communications, 1989,25(5):77-78.
[4] Q.D.Su,Q.Wang,Y.T.Yang, Plant Physiology Communications, 1996,03:206-207.

[5] F.J.Xu, Analytical Laboratory, 2005,09:65-89.

[6] T.C.Ai,F.M.Li,Z.A.Zhou, Journal of HuBei Agricultural College, 2000,20(1):6-8.

[7] W.Y.Zhang,K.H.Wang, Chinese Agricultural Science Bulletin, 2012,28(21):92-95

[8] J.Q.Guo,H.M.Pu, J.F. Zhang,W.H.Long, Agricultural Science \& Technology, 2013, 14(10): 1421-1428

[9] Y.1.YU,J.W.Kai,C.H.Wang,J.B.Wen, Journal of Maize Sciences, 2011,9(4): 89 92, 97

[10] P.C. Li, H.L.Dong, A.Z.Liu, R.Z.Wang, Chinese Agricultural Science Bulletin, 2012,28(3):121-126.

[11] Y.P.Lin, J.H.Jin, Chemical Analysis and Meterage, 2004,13(3):52-55.

[12] Z.Y. Wang, D.S.Chen, L. Huang, Transactions of the Chinese Society of Agricultural Engineering, 2001.17(3):142-144.

[13] Frick J,Precetti C,Mitchell ,Am. Soc. Horticul. Sci., (1998).123: 1076-1080. 